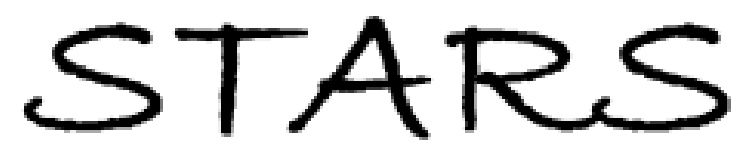

University of Central Florida

STARS

$1-1-2006$

\title{
Thermomechanical oscillations in hybrid nematic liquid crystals
}

\author{
R. S. Hakobyan \\ G. L. Yesayan \\ B. Ya. Zeldovich \\ University of Central Florida
}

Find similar works at: https://stars.library.ucf.edu/facultybib2000 University of Central Florida Libraries http://library.ucf.edu

This Article is brought to you for free and open access by the Faculty Bibliography at STARS. It has been accepted for inclusion in Faculty Bibliography 2000s by an authorized administrator of STARS. For more information, please contactSTARS@ucf.edu.

\section{Recommended Citation}

Hakobyan, R. S.; Yesayan, G. L.; and Zeldovich, B. Ya., "Thermomechanical oscillations in hybrid nematic liquid crystals" (2006). Faculty Bibliography 2000s. 6192.

https://stars.library.ucf.edu/facultybib2000/6192

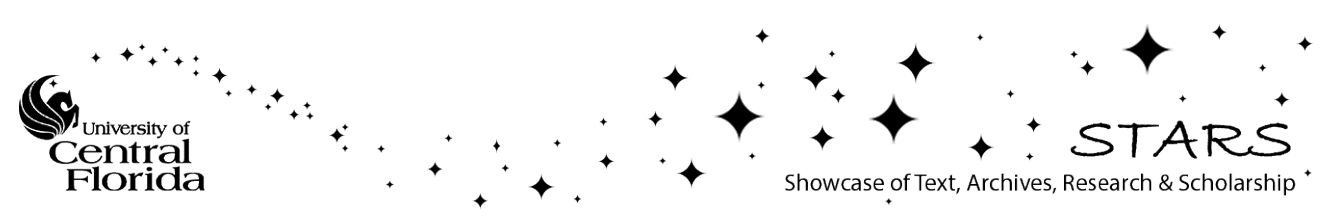




\title{
Thermomechanical oscillations in hybrid nematic liquid crystals
}

\author{
R. S. Hakobyan and G. L. Yesayan \\ Department of Physics, Yerevan State University, 375049, Yerevan, Armenia \\ B. Ya. Zeldovich \\ CREOL College of Optics and Photonics, University of Central Florida, P.O. Box 16-2700, Orlando, Florida 32816-2700, USA
}

(Received 12 May 2005; revised manuscript received 2 November 2005; published 15 June 2006)

\begin{abstract}
Thermomechanical oscillations observed previously in hybrid oriented nematic liquid crystals (NLCs) are explained theoretically. The effect is conditioned by the hydrodynamic flow tendency to reduce the curvature of the "flexible ribbon" of hybrid NLCs. There is qualitative agreement between the theoretical calculations and the experimental results.
\end{abstract}

DOI: 10.1103/PhysRevE.73.061707

PACS number(s): $61.30 . \mathrm{Gd}, 65.40 . \mathrm{De}, 42.50 . \mathrm{Vk}$

\section{INTRODUCTION}

The thermomechanical effect in gases [1] and superfluid liquids [2] is well known. The thermomechanical rotation in a cholesteric liquid crystal due to the vertical temperature gradient was known long time ago and investigated in detail in [3-7]. This effect is uniquely related to the chirality of cholesterics, i.e., to the fact that they have no right-handleft-hand symmetry. It has been supposed that these effects have no analogs in nonchiral liquid crystals (LCs), particularly for nematics $[4,8]$.

Nevertheless, a number of thermomechanical effects for nonchiral LCs, namely for nematics, were predicted in [9]. Thermomechanical effects of three basic types were considered: Hydrodynamic flow excitation induced by temperature gradient (direct thermomechanical effect), temperature gradient arising in nonuniform flow (inverse thermomechanical effect), and additional director deflection caused by heat flow. An expression for the "dissipation" function was obtained. Variation of this function makes possible a description of the foregoing effects. The same terms in the thermomechanical equations were written in [10]. The first experimental observation of the rotation of nematic material due to a temperature gradient was carried out with a nematic drop [11]. The study of the dependence of the flow speed versus sample thickness allowed the authors [9] to conclude that this effect corresponds to the thermomechanical effect predicted earlier. The experimentally estimated thermomechanical coupling constant was in good agreement with the theoretical calculations [9]. Recently, the thermomechanical effect in hybrid and cylindrical-hybrid-oriented horizontal layers of nematic LCs (NLCs) due to a vertical temperature gradient [12] has been investigated experimentally and theoretically. The essence of the effect is the formation of a horizontal hydrodynamic flow in the NLC with an inhomogeneous director distribution. In the case when the hydrodynamic flow tends to reduce the curvature of the "flexible ribbon", an oscillatory motion was observed.

In this work, we investigate theoretically the possibility of hybrid curvature reversal due to the hydrodynamic flow. This model allows us to explain our previous experimental result about oscillatory hydrodynamic flow due to the thermomechanical forces at the presence of a transverse temperature gradient.
The paper is organized as follows. In Sec. II, we discuss the equations describing the thermomechanical effect in hybrid-oriented nematics. In Sec. III, we investigate the possibility of hybrid curvature reversal due to the hydrodynamic flow. Section IV contains the description of thermomechanical oscillation in hybrid-oriented NLCs. Finally, we give a summary of the conclusions in Sec. V.

\section{THERMOMECHANICAL EQUATIONS IN A HYBRID NLC}

Let us consider an NLC cell with the so-called hybrid orientation (Fig. 1). We direct the normal to the cell walls along the $z$ axis and assume that the boundary condition on the wall specifies homeotropic orientation $\vec{n}(z=0)=\vec{e}_{z}$ at $z$ $=0$ and planar orientation $\vec{n}(z=L)=\vec{e}_{x}$ at $z=L$, where $\vec{n}$ is the director unit vector, with $\vec{n}$ and $-\vec{n}$ equivalent, and $L$ is the cell thickness. Let the external heat sources maintain temperature $T=T_{0}$ at the plane $z=0$ and temperature $T=T_{0}+\Delta T$ at the plane $z=L$. The temperature gradient $d T / d z \sim \Delta T / L$ leads then, according to [9], to tangential thermomechanical stresses $\sigma_{z x}^{\text {thm }} \approx \xi \Delta T / L^{2}$, where $\xi$ is the thermomechanical constant. The result is liquid flow in the $x$ direction. The stationary velocity $v$ in this flow can be roughly estimated by equating the thermomechanical and Navier-Stokes contributions in the stress tensor $\sigma_{i k}$. Assuming for the latter $\sigma_{z x}^{\prime}$ $\approx \eta v / L$, where $\eta$ is viscosity, we obtain $v \approx \xi \Delta T / L \eta$. This hydrodynamic flow leads to the reorientation of the NLC director. The direction of the flow velocity depends on the director concavity and the temperature gradient direction. If the temperature gradient is directed from the cell wall with a

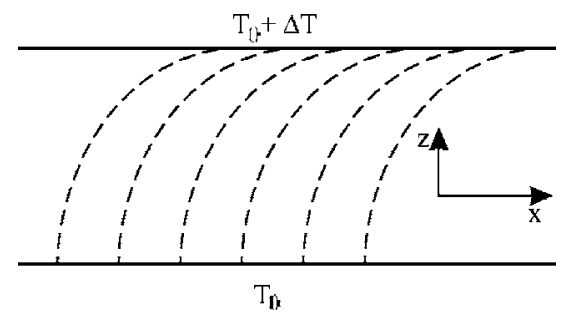

FIG. 1. Director profile in a nematic LC cell with homogeneous temperature. 
planar boundary condition to the wall with a homeotropic condition, then thermomechanical hydrodynamical flow tends to increase the curvature of the flexible ribbon of hybrid NLC. If the temperature gradient has the opposite direction, then flow tends to reverse the curvature of the flexible ribbon. In the later case, thermomechanical stress induced the oscillatory hydrodynamical flow.

In order to describe the above-mentioned thermomechanical effects, we need to write equations of nematodynamics taking into account the thermomechanical stress tensor. They are balance equations of torque acting on the NLC director and the Navier-Stokes equation.

Torque balance equations can be obtained from the variation principle [13]:

$$
[\vec{f} \times \vec{n}]_{i}+e_{i j m} n_{m}\left[\frac{\delta F}{\delta n_{i}}-\frac{\partial}{\partial x_{k}} \frac{\delta F}{\delta\left(\partial n_{j} / \partial x_{k}\right)}\right]=0,
$$

where $e_{i j m}$ is the whole antisymmetric tensor, $\vec{f}$ is the hydrodynamical "force" acting on the NLC director and expressed through the generalized velocities $\vec{N}$, and the velocitygradient tensor $d_{i j}$ :

$$
\begin{gathered}
f_{i}=\left(\alpha_{3}-\alpha_{2}\right) N_{i}+\left(\alpha_{3}+\alpha_{2}\right) d_{i j} n_{j}, \\
N_{i}=\frac{d n_{i}}{d t}+\frac{1}{2}(\vec{n} \times \operatorname{curl} \vec{v})_{i}, \quad d_{i j}=\frac{1}{2}\left(\frac{\partial v_{i}}{\partial x_{j}}+\frac{\partial v_{j}}{\partial x_{i}}\right) .
\end{gathered}
$$

Here, $F$ is the free-energy density in its usual Frank's form:

$$
F=\frac{1}{2} K_{1}(\operatorname{div} \vec{n})^{2}+\frac{1}{2} K_{2}(\vec{n} \cdot \operatorname{curl} \vec{n})^{2}+\frac{1}{2} K_{3}(\vec{n} \times \operatorname{curl} \vec{n})^{2},
$$

where $K_{i}$ are Frank's elastic constants, and $\alpha_{i}$ are Leslie coefficients of the NLC. The Navier-Stokes equation for hydrodynamic flow velocity $\vec{v}(\vec{r}, t)$ of an incompressible NLC, with the presence of thermomechanical terms, is of the form

$$
\begin{gathered}
\rho\left(\frac{\partial v_{i}}{\partial t}+(\vec{v} \cdot \vec{\nabla}) v_{i}\right)=\frac{\partial \sigma_{k i}}{\partial x_{k}}, \\
\sigma_{k i}=-p \delta_{k i}+\sigma_{k i}^{\prime}+\sigma_{k i}^{\mathrm{thm}}, \quad \operatorname{div} \vec{v}=0,
\end{gathered}
$$

where $\rho$ is the density, $p(\vec{r}, t)$ is the hydrodynamic pressure determined from the same set of Eqs. (5) and (6) and the boundary conditions, $\sigma_{k i}^{\prime}$ is the viscous stress tensor, and $\sigma_{k i}^{\text {thm }}$ is the thermomechanical stress tensor [9].

We consider the problem homogeneous in the $(x, y)$ plane $(\partial / \partial x=\partial / \partial y=0)$ and the director distribution in the $(x, z)$ plane $\left(n_{y}=0\right)$. We have the boundary conditions for $\vec{n}$ mentioned above. Thermomechanical stress leads to the hydrodynamic flow with velocity $\vec{v}$ directed in the $x$ direction $(\vec{v}$ $\left.=\vec{e}_{x} v\right)$. For this problem, Eq. (1) for director $\vec{n}=\left(n_{x}, 0, n_{z}\right)$ has the form

$$
\begin{aligned}
\left(\alpha_{3}-\right. & \left.\alpha_{2}\right)\left(n_{x} \frac{\partial n_{z}}{\partial t}-n_{z} \frac{\partial n_{x}}{\partial t}\right)+\left(\alpha_{3} n_{x}^{2}-\alpha_{2} n_{z}^{2}\right) \frac{\partial v}{\partial z}+K_{3} n_{z} \frac{\partial^{2} n_{x}}{\partial z^{2}} \\
- & K_{1} n_{x} \frac{\partial^{2} n_{z}}{\partial z^{2}}=0 .
\end{aligned}
$$

Equation (7) describes reorientation of the NLC director under the influence of the hydrodynamic velocity gradient. We are going to assume, based on some good rationale [13], that the inverse influence of the anisotropy on the velocity can be neglected in our problem. Therefore, the viscous stress tensor has the isotropic form: $\sigma_{k i}^{\prime}=0.5 \alpha_{4}\left(\partial v_{i} / \partial x_{k}+\partial v_{k} / \partial x_{i}\right)$. And, for the velocity in the thermomechanical single-constant approximation $\left(\xi_{1}=\xi_{2}=\ldots=\xi_{12}=\xi\right)$, we have

$$
\rho \frac{\partial v}{\partial t}=\eta \frac{\partial^{2} v}{\partial z^{2}}+\frac{1}{4} \xi \frac{d T}{d z}\left[\left(5+3 n_{x}^{2}\right) \frac{\partial n_{x}}{\partial z} \frac{\partial n_{z}}{\partial z}+\left(5 n_{x}+n_{x}^{3}\right) \frac{\partial^{2} n_{z}}{\partial z^{2}}\right] .
$$

Equations (7) and (8) are generalized equations for the description of thermomechanical flow in an NLC with the director confined to the $(x, z)$ plane.

\section{HYBRID NLC DIRECTOR INSTABILITY AT THE PRESENCE OF SHEAR FLOW}

We denote the angle between the director and $x$ axis as $\theta(z, t)$; then $n_{x}=\sin \theta(z, t), n_{z}=\cos \theta(z, t)$, and the equation for the orientation angle has the form

$$
\begin{aligned}
\left(\alpha_{3}-\alpha_{2}\right) \frac{\partial \theta}{\partial t}= & \left(K_{1} \sin ^{2} \theta+K_{3} \cos ^{2} \theta\right) \frac{\partial^{2} \theta}{\partial z^{2}} \\
& +\left(K_{1}-K_{3}\right) \sin \theta \cos \theta\left(\frac{\partial \theta}{\partial z}\right)^{2} \\
& +\left(\alpha_{3} \sin ^{2} \theta-\alpha_{2} \cos ^{2} \theta\right) \frac{\partial v}{\partial z} .
\end{aligned}
$$

Let us consider first the behavior of the hybrid NLC director in the presence of simple hydrodynamic flow; shear flow as an example $\left(v=v_{0} z / L, v_{0}=\right.$ const $)$. The boundary conditions for the orientational angle are: $\theta(z=0, t)=0$ and $\theta(z=L, t)$ $= \pm \pi / 2$ (" + " if the flexible ribbon is oriented in the positive direction of $x$, and "-" otherwise). In the absence of flow, the stationary solution of Eq. (9) with mentioned boundary conditions has, in good approximation, linear profile: $\theta(z)$ $= \pm \pi z /(2 L)$. Thus, we can assume this solution as an initial condition for the general problem. In the general case, for the finite anchoring energy and under the Rapini approximation, the boundary conditions have the following form [14]:

$$
\left(K_{1} \sin ^{2} \theta+K_{3} \cos ^{2} \theta\right) \frac{\partial \theta}{\partial z}-\sigma_{1} \sin \theta \cos \theta=0
$$

at lower wall $(z=0)$ and at upper wall $(z=L)$ :

$$
\left(K_{1} \sin ^{2} \theta+K_{3} \cos ^{2} \theta\right) \frac{\partial \theta}{\partial z}-\sigma_{2} \sin \theta \cos \theta=0 .
$$

Let us note that Equation (9) takes the form of the wellknown "damped-driven sine-Gordon equation" in the single- 

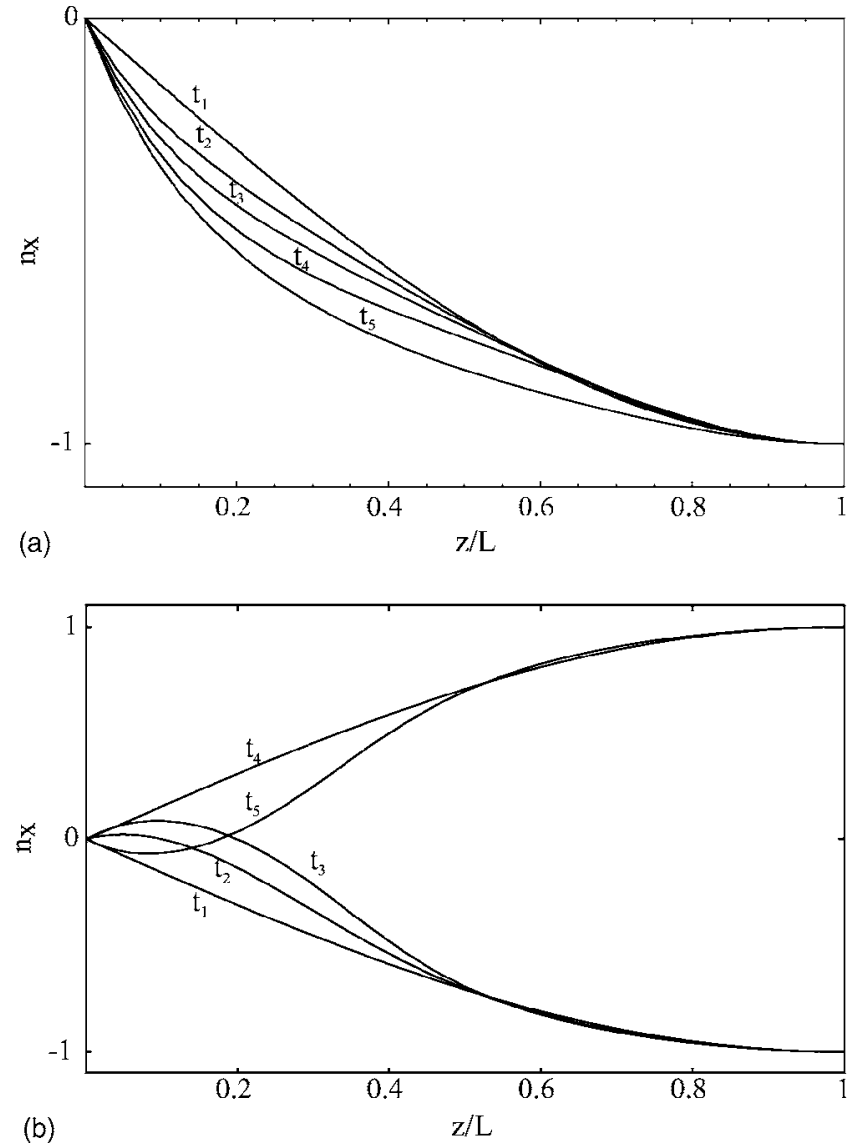

FIG. 2. Profiles of the director's $x$ component $\left(n_{x}\right)$ for different times $\left(t_{j}\right)$ after switching the temperature gradient "on": (a) The temperature gradient is directed from planar boundary to homeotropic one $\left(t_{1}=0, t_{2}=0.39 \mathrm{~s}, t_{3}=0.77 \mathrm{~s}, t_{4}=1.55 \mathrm{~s}\right.$, and $\left.t_{5}=7.74 \mathrm{~s}\right)$; and (b) the temperature gradient is directed from homeotropic boundary to planar one $\left(t_{1}=0, t_{2}=0.77 \mathrm{~s}, t_{3}=1.55 \mathrm{~s}, t_{4}=3.35 \mathrm{~s}\right.$, and $t_{5}$ $=4.64 \mathrm{~s})$. Reversal time is $t_{r}=3.35 \mathrm{~s}$ and oscillation period is $t_{p}$ $=6.7 \mathrm{~s}$.

constant approximation for elastic constants $\left(K_{1}=K_{3}\right)$.

We were able to solve Eq. (9) for director reorientation under the influence of flow with the above-mentioned boundary and initial conditions using MATHEMATICA-5. In this calculation for NLC MBBA, we assumed $K_{1}=6$ $\times 10^{-7} \mathrm{erg} / \mathrm{cm} ; \quad K_{3}=7.5 \times 10^{-7} \mathrm{erg} / \mathrm{cm} ; \alpha_{2}=-0.77 P$; and $\alpha_{3}=-0.012 P$. If hydrodynamic flow velocity is directed out of the flexible ribbons' curvature (in the opposite direction of $x$ axis in Fig. 1), then the velocity gradient brings about a small increase of curvature [see Fig. 2(a)]. The curvature deforms more completely and the deformation increases in time when velocity is directed into (in the direction of $x$ axis) the flexible ribbons' curvature [Fig. 2(b)]. Thereby, the director deformation elastic energy $F$ increases in time, where

$$
F=\frac{1}{2} K_{1}\left(\frac{\partial n_{z}}{\partial z}\right)^{2}+\frac{1}{2} K_{3}\left(\frac{\partial n_{x}}{\partial z}\right)^{2} .
$$

The flexible ribbon reverses its curvature at the time when deformation energy becomes larger than surface anchoring energy at $z=L$. In that way, velocity is directed out of the reversed curvature. The reversing time depends on the parameters and surface coupling energy of the NLC. Later, it depends on the method of surface treatment.

\section{THERMOMECHANICAL OSCILLATION IN HYBRID NLC}

Now let us consider the thermomechanical effect in a hybrid-oriented nematic with initial director distribution $\theta(z, t=0)= \pm \pi z / 2 L$. Let the condition $T(z=0)>T(z=L)$ be satisfied for the temperatures kept on the NLC cell substrates. Then, in the presence of the gradient of the director orientation angle along the $z$ coordinate, a thermomechanical hydrodynamic flow appears, which moves along the $x$ axis at the velocity $v$. When we have initial condition $\theta(z, t=0)$ $=\pi z / 2 L$, the flexible ribbon convexity is directed opposite to the positive $x$ direction (as in Fig. 1). The thermomechanical hydrodynamical flow is directed in the same way, out of the flexible ribbons' curvature, and causes a little deepening of the curvature. When we have the initial condition $\theta(z, t=0)$ $=-\pi z / 2 L$, the flexible ribbon convexity is directed along the positive $x$ direction. The thermomechanical hydrodynamical flow is directed in the same way, and again causes some deepening of curvature.

Let us heat the same cell from the wall with $z=L: T(z$ $=0)<T(z=L)$. Then, for both initial conditions, the thermomechanical hydrodynamical flow is directed into flexible ribbons' curvature and deforms it completely. This deformation increases in time and induces the reversal of curvature. This leads to velocity reversal. That is why in this reverse case of curvature and velocity, thermomechanical velocity again has the direction into the flexible ribbons' curvature and so it again causes a reversal of curvature and velocity. Thereby, we can observe oscillatory thermomechanical motion; the period of which is equal to twice the time of the first reversal of curvature or velocity. This oscillatory behavior of NLC director orientation and thermomechanical hydrodynamical velocity can be described by the system of Eqs. (8) and (9). The boundary conditions for velocity are $v(z=0, t)=v(z$ $=L, t)=0$ and the initial condition is $v(z, t=0)=0$. For calculation with MATHEMATICA-5, we assume for NLC MBBA $\rho$ $=1 \mathrm{~g} / \mathrm{cm}^{3}, \quad \xi=10^{-6} \mathrm{erg} / \mathrm{cm} \mathrm{K}, \Delta T=10 \mathrm{~K}$, and with cell thickness $L=10^{-2} \mathrm{~cm}$. Velocity starts to grow from zero with establishment time on six orders shorter than for the director. That is why in our model of flow and director reversing the dynamic of the fluid particles, braking and acceleration near the points of flow reversal are ignored. And, for velocity, we have only the change of sign. The spatial $(Z=z / L)$ distribution of velocity was found to be nearly parabolic. For the velocity magnitude, we have $v \approx 10^{-3} \mathrm{~cm} / \mathrm{s}$. As we have mentioned above, surface anchoring energy depends on the method of surface treatment. It can be several times larger than the deformation free-energy $F$ for a stationary distribution of the NLC director. In our calculation, we will take it as twice larger than the deformation energy for initial stationary distribution. In Fig. 3, we have plotted the time $\left\{\tau=\left[K_{1} /\left(\alpha_{3}-\alpha_{2}\right) L^{2}\right] t\right\}$ and spatial $(Z=z / L)$ dependence of the $x$ component of the director unit vector $n_{x}$. A reversal from the first solution to the second is seen. As we have 


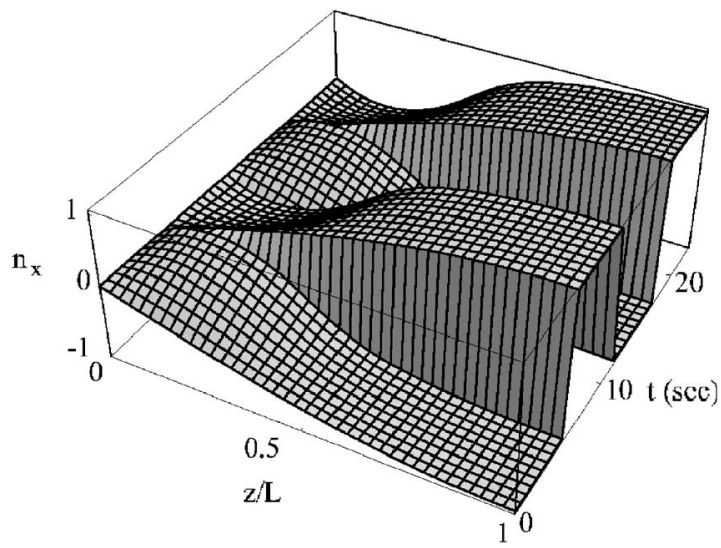

FIG. 3. Three-dimensional time and space dependence of the director's $x$ component after switching the temperature gradient on; temperature gradient is directed from homeotropic boundary to planar one.

mentioned above, the period of oscillation is twice that of the reversal time. So, for the cell thickness $L=10^{-2} \mathrm{~cm}$, we obtain a period of $\tau_{p}=6.7 \mathrm{~s}$. The oscillation period strongly depends on the surface coupling energy. The hybrid NLC director distribution cannot exist when the surface coupling energy is less than the free energy of initial stationary director's distribution (in our case, it was $1.25 \times 10^{-2}$ erg per $\mathrm{cm}^{2}$ of cell surface). This oscillation period strongly increases with an increase in the surface coupling energy from its minimal value (see Fig. 4).

It is well known that the Raleigh-Benard convective instability may develop in a horizontal NLC cell in the presence of vertical temperature gradient [15]. This effect has threshold character. On the contrary, thermomechanical reorientation has no threshold behavior. In the case with $\Delta T$ $=10 \mathrm{~K}, L=10^{-2} \mathrm{~cm}$, and for NLC MBBA, we have very high director reorientation due to our thermomechanical effect, while the threshold of convective instability is much larger $\left(\Delta T=10^{3} \mathrm{~K}\right.$ for $\left.L=10^{-2} \mathrm{~cm}\right)$.

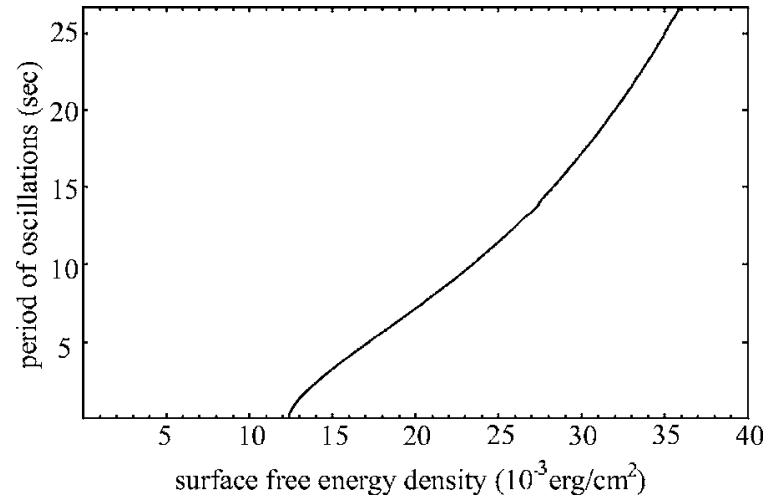

FIG. 4. Surface free energy per $\mathrm{cm}^{2}$ of the LC cell plane respect to thermomechanical oscillation period.

It is important to note that we have actually varied different parameters within the factor range from 0.5 to 2 . No qualitative changes in the behavior of the system were observed in our modeling.

\section{DISCUSSION}

Our analysis and numerical estimates are in qualitative agreement with experimental results in our previous study [12]. An investigation of these effects may, in our opinion, yield new important information on the molecular dynamics of the mesophase of LCs. Beside the effect considered in this paper (which is connected with temperature gradients), other effects should occur in LCs: Electromechanical effect due to electric-field gradients, and diffusion-mechanical effect related to density gradients of some component or impurity.

\section{ACKNOWLEDGMENTS}

This research was made possible in part by Award No. AP2-2302-YE-02 of the U.S. Civilian Research and Development Foundation for the Independent States of the Former Soviet Union (CRDF). The authors are grateful to Kirk H. Michaelian for useful discussions.
[1] E. M. Lifshitz and L. P. Pitaevskii, Physical Kinetics (Butterworth-Heinemann; Reprint edition, 1981).

[2] L. D. Landau and E. M. Lifshitz, Fluid Mechanics (Pergamon, Oxford, 1987).

[3] O. Lehmann, Ann. Phys. 4, 649 (1900).

[4] F. M. Leslie, Proc. R. Soc. London, Ser. A 307, 359 (1968).

[5] V. Janossy, Mol. Cryst. Liq. Cryst. Lett. 72, 233 (1982).

[6] G. S. Ranganath, Mol. Cryst. Liq. Cryst. 92, 105 (1983).

[7] M. Leslie, J. Non-Equil. Thermodyn. 11, 23 (1986).

[8] S. Chandrasekhar, Liquid Crystals (Cambridge University Press, Cambridge, UK, 1980).

[9] R. S. Akopyan and B. Y. Zel'dovich, Sov. Phys. JETP 60, 953
(1984).

[10] H. R. Brand and H. Pleiner, Phys. Rev. A 35, 3122 (1987).

[11] O. D. Lavrentovich and Y. A. Nastishin, Ukr. Fiz. Zh. (Russ. Ed.) 32, 710 (1987).

[12] R. S. Akopyan, R. B. Alaverdian, E. A. Santrosian, and Y. S. Chilingarian, J. Appl. Phys. 90, 3371 (2001).

[13] R. S. Akopyan and B. Y. Zel'dovich, Sov. Phys. JETP 59, 311 (1984).

[14] F. Simoni, Nonlinear Optical Properties of Liquid Crystals and PDLC (World Scientific, Teaneck, New Jersey, 1997).

[15] E. Guyon, P. Pieranski, and M. A. Guinier, C. R. Seances Acad. Sci., Ser. B 274, 656 (1972). 